\title{
Computing the Grothendieck constant of some graph classes
}

\author{
M. Laurent ${ }^{\mathrm{a}, \mathrm{b}}, \mathrm{A}$. Varvitsiotis ${ }^{\mathrm{a}, *}$ \\ ${ }^{a}$ Centrum Wiskunde 85 Informatica (CWI), Science Park 123, 1098 XG Amsterdam, The Netherlands. \\ ${ }^{b}$ Tilburg University, P.O. Box 90153, 5000 LE Tilburg, The Netherlands.
}

\begin{abstract}
Given a graph $G=([n], E)$ and $w \in \mathbb{R}^{E}$, consider the integer program $\max _{x \in\{ \pm 1\}^{n}} \sum_{i j \in E} w_{i j} x_{i} x_{j}$ and its canonical semidefinite programming relaxation $\max \sum_{i j \in E} w_{i j} v_{i}^{T} v_{j}$, where the maximum is taken over all unit vectors $v_{i} \in \mathbb{R}^{n}$. The integrality gap of this relaxation is known as the Grothendieck constant $\kappa(G)$ of $G$. We present a closed-form formula for the Grothendieck constant of $K_{5}$-minor free graphs and derive that it is at most 3/2. Moreover, we show that $\kappa(G) \leq \kappa\left(K_{k}\right)$ if the cut polytope of $G$ is defined by inequalities supported by at most $k$ points. Lastly, since the Grothendieck constant of $K_{n}$ grows as $\Theta(\log n)$, it is interesting to identify instances with large gap. However this is not the case for the clique-web inequalities, a wide class of valid inequalities for the cut polytope, whose integrality ratio is shown to be bounded by 3 .
\end{abstract}

Keywords: Grothendieck constant, elliptope, cut polytope, clique-web inequality

\section{Introduction}

Let $G=([n], E)$ be a (simple loopless) graph and $w=\left(w_{i j}\right) \in \mathbb{R}^{E}$. Consider the integer quadratic program over the hypercube

$$
\operatorname{ip}(G, w):=\max _{x \in\{ \pm 1\}^{n}} \sum_{i j \in E} w_{i j} x_{i} x_{j}
$$

and its canonical semidefinite programming relaxation

$$
\operatorname{sdp}(G, w):=\max _{u_{1}, \ldots, u_{n} \in \mathbb{R}^{n},\left\|u_{i}\right\|=1} \sum_{i j \in E} w_{i j} u_{i}^{T} u_{j} .
$$

Let $\kappa(G)$ denote the integrality gap of relaxation (2), defined by

$$
\kappa(G)=\sup _{w \in \mathbb{R}^{E}} \frac{\operatorname{sdp}(G, w)}{\operatorname{ip}(G, w)} .
$$

In other words, $\kappa(G)$ is the smallest constant $K>0$ for which $\operatorname{sdp}(G, w) \leq K \cdot \operatorname{ip}(G, w), \forall w \in \mathbb{R}^{E}$. Alon et al. 1] call this graph parameter the Grothendieck constant of $G$ and prove that

$$
\Omega(\log \omega(G))=\kappa(G)=O(\log \vartheta(\bar{G})) .
$$

*Corresponding author: CWI, Postbus 94079, 1090 GB Amsterdam. Tel: +31 20 5924170; Fax: +31 205924199 .

Email addresses: M.Laurent@cwi.nl (M. Laurent),

A.Varvitsiotis@cwi.nl (A. Varvitsiotis)
Here $\omega(G)$ denotes the maximum size of a clique in $G$ and $\vartheta(\bar{G})$ the Lovász theta function of the complementary graph $\bar{G}$, for which it is known that $\omega(G) \leq \vartheta(\bar{G}) \leq \chi(G)[16$. Hence, for the complete graph $G=K_{n}, \kappa\left(K_{n}\right)=\Theta(\log n)$.

The name of the constant goes back to Grothendieck [12], who considered the case of bipartite graphs and showed the existence of a constant $K>0$ for which $\operatorname{sdp}\left(K_{m, n}, w\right) \leq K \cdot \operatorname{ip}\left(K_{m, n}, w\right)$ for all $m, n \in \mathbb{N}$ and $w \in \mathbb{R}^{m n}$. The smallest such constant is known as Grothendieck's constant and is denoted by $K_{G}$. It is a long standing open problem to compute the exact value of $K_{G}$. It is known that $K_{G}<\pi[2 \ln (1+\sqrt{2})]^{-1} \sim 1.782[13$, 7], and that $K_{G} \geq 1.6769 \ldots$ 20]. Recently Briët et al. [8] show that $\kappa(G) \leq \frac{\pi}{2 \operatorname{arcsinh}\left((\vartheta(G)-1)^{-1}\right)}$, which gives the above bound 1.782 for bipartite graphs and improves the upper bound in (4) when $\vartheta(\bar{G})$ is small.

In recent years, Grothendieck type inequalities have received a significant amount of attention due to their various applications, most notably in the design of approximation algorithms and quantum information theory (see, e.g., [1, 2, 7, 10, 17, 22]).

The paper is organized as follows. In Section 2 we collect basic properties of $\kappa(G)$. In Section 3 we establish a closed-form formula for the Grothendieck constant of $K_{5}$-minor free graphs in terms of their girth and bound $\kappa(G)$ in terms of the size of the 
supports of the facets of the cut polytope. In Section 4 we show that the integrality gap achieved by the clique-web inequalities, a wide class of valid inequalities for the cut-polytope, is bounded by 3 .

\section{Basic properties}

We first introduce some notation. Throughout $[n]=\{1, \ldots, n\}$. Let $\mathcal{S}_{n}^{+}$denote the cone of positive semidefinite matrices; the notation $A \succeq 0$ means that $A \in \mathcal{S}_{n}^{+}$. For matrices $A, B,\langle A, B\rangle=$ $\sum_{i, j} A_{i j} B_{i j}$ stands for the usual trace inner product. Let $e$ denote the all ones vector and $J=e e^{T}$ the all ones matrix, of the appropriate dimension.

Let $\mathcal{E}_{n}:=\left\{X \in \mathcal{S}_{n}^{+} \mid X_{i i}=1 \forall i \in[n]\right\}$ and $\mathrm{CUT}_{n}:=\operatorname{conv}\left(X \in \mathcal{E}_{n} \mid \operatorname{rank} X=1\right\}$. Moreover, define $\mathcal{E}(G):=\pi_{E}\left(\mathcal{E}_{n}\right), \operatorname{CUT}(G):=\pi_{E}\left(\mathrm{CUT}_{n}\right)$, where $\pi_{E}$ denotes the projection from $\mathbb{R}^{n \times n}$ onto the subspace $\mathbb{R}^{E}$ indexed by the edge set of $G$. They are known, respectively, as the elliptope and the cut polytope of $G$ and satisfy $\operatorname{CUT}(G) \subseteq \mathcal{E}(G)$. We refer, e.g., to [9] and further references therein for a detailed study of these geometric objects.

For $w \in \mathbb{R}^{E}$, let $\kappa(G, w)=\operatorname{sdp}(G, w) / \operatorname{ip}(G, w)$.

\subsection{A geometric reformulation for $\kappa(G)$}

Clearly, the Grothendieck constant $\kappa(G)$ is the smallest dilation of $\operatorname{CUT}(G)$ containing $\mathcal{E}(G)$.

Lemma 2.1. For any graph $G$,

$$
\kappa(G)=\min \{K \mid \mathcal{E}(G) \subseteq K \cdot \operatorname{CUT}(G)\} .
$$

Proof. Directly, since $\operatorname{ip}(G, w)=\max _{x \in \operatorname{CUT}(G)} w^{T} x$ and $\operatorname{sdp}(G, w)=\max _{x \in \mathcal{E}(G)} w^{T} x$.

As the origin lies in the interior of $\operatorname{CUT}(G)$, the polytope $\operatorname{CUT}(G)$ has a linear inequality description consisting of finitely many facet-defining inequalities of the form $w^{T} x \leq 1$. Let us recall the following switching operation: Given $w \in \mathbb{R}^{E}$, its switching by $S \subseteq[n]$ is the vector $w^{(S)} \in \mathbb{R}^{E}$ whose $(i, j)$-th entry is $-w_{i j}$ if the edge $i j$ is cut by the partition $(S,[n] \backslash S)$ and $w_{i j}$ otherwise. It is well known that the switching operation preserves valid inequalities and facet defining inequalities of the cut polytope [5, 9]. Moreover, $\operatorname{sdp}(G, w)=$ $\operatorname{sdp}\left(G, w^{(S)}\right)$ and $\operatorname{ip}(G, w)=\operatorname{ip}\left(G, w^{(S)}\right)$. This implies the next lemma which gives a useful reformulation for $\kappa(G)$.
Lemma 2.2. For any graph $G$,

$$
\kappa(G)=\sup _{w \in \mathbb{R}^{E}} \kappa(G, w),
$$

where the supremum ranges over all facet defining inequalities of $\operatorname{CUT}(G)$, distinct up to switching.

\subsection{Connections with max-cut}

The study of the cut polytope $\operatorname{CUT}(G)$ and of the elliptope $\mathcal{E}(G)$ is largely motivated by their relevance to the maximum cut problem in combinatorial optimization. Given $G=([n], E)$ and $w \in \mathbb{R}^{E}$, the max-cut problem asks for a cut of maximum weight. Thus we want to compute $\operatorname{mc}(G, w)=\max _{x \in\{ \pm 1\}^{n}} \frac{1}{2} \sum_{i j \in E} w_{i j}\left(1-x_{i} x_{j}\right)=$ $\max _{x \in\{ \pm 1\}^{n}} \frac{1}{4} x^{T} L_{G, w} x$. Here, $L_{G, w}$ is the Laplacian matrix, with $(i, i)$-th entry $\sum_{j} w_{i j}$ and with $(i, j)$-th entry $-w_{i j}$ if $i j \in E$ and 0 otherwise. The canonical semidefinite programming relaxation of max-cut (considered e.g. in [11]) is $\operatorname{sdp}_{\mathrm{GW}}(G, w)=\max _{X \in \mathcal{E}_{n}} \frac{1}{4}\left\langle L_{G, w}, X\right\rangle$. Hence the quadratic integer problem (1) and the max-cut problem are affine transforms of each other, and the same for their canonical semidefinite relaxations; namely, $\operatorname{mc}(G, w)=\frac{1}{2}(w(E)+\operatorname{ip}(G,-w))$ and $\operatorname{sdp}_{\mathrm{GW}}(G, w)=\frac{1}{2}(w(E)+\operatorname{sdp}(G,-w))$.

In particular, this implies that, given $w \in \mathbb{Q}^{E}$, deciding whether $\operatorname{ip}(G, w)=\operatorname{sdp}(G, w)$ is an NPcomplete problem [18].

The following lemma is easy to verify.

Lemma 2.3. Let $A \in \mathcal{S}_{n}^{+}$and $B=\left(\begin{array}{cc}0 & A / 2 \\ A / 2 & 0\end{array}\right)$.

Then, $\max _{Z \in \mathcal{E}_{2 n}}\langle B, Z\rangle=\max _{X \in \mathcal{E}_{n}}\langle A, X\rangle$, and $\max _{z \in\{ \pm 1\}^{2 n}} z^{T} B z=\max _{x \in\{ \pm 1\}^{n}} x^{T} A x$.

When $w \geq 0, L_{\mathrm{G}, w} \succeq 0$ and thus Lemma 2.3 implies that $\operatorname{sdp}_{\mathrm{GW}}(G, w)=\max _{Z \in \mathcal{E}_{2 n}}\langle B, Z\rangle$, where $B$ is as in the lemma with $A / 2:=L_{G, w} / 8$. By the definition of the Grothendieck constant $K_{G}$, this implies that $\operatorname{sdp}_{\mathrm{GW}}(G, w) \leq K_{G} \cdot \operatorname{mc}(G, w)$. However, this approximation guarantee is not interesting since we know by [11] that $\operatorname{sdp}_{\mathrm{GW}}(G, w) \leq 1.138 \cdot \operatorname{mc}(G, w)$, while $K_{G} \geq 1.6$.

On the other hand, the Grothendieck constant $\kappa(G)$ bounds the semidefinite approximation for max-cut for edge weights satisfying $w(E) \geq 0$.

Lemma 2.4. Let $G=(V, E)$ be a graph and $w \in$ $\mathbb{R}^{E}$ with $w(E) \geq 0$ and $\operatorname{mc}(G, w)>0$. Then, $\operatorname{sdp}_{G W}(G, w) \leq \kappa(G) \cdot \operatorname{mc}(G, w)$. 
Proof. Indeed, $\operatorname{sdp}(G,-w) \leq \kappa(G) \cdot \operatorname{ip}(G,-w)$ and $w(E) \leq \kappa(G) \cdot w(E)$ imply

$$
\frac{\operatorname{sdp}_{\mathrm{GW}}(G, w)}{\operatorname{mc}(G, w)}=\frac{w(E)+\operatorname{sdp}(G,-w)}{w(E)+\operatorname{ip}(G,-w)} \leq \kappa(G) .
$$

\subsection{Behaviour under graph operations}

It follows immediately from the definition that the graph parameter $\kappa(\cdot)$ is monotone nonincreasing with respect to deleting edges. That is,

Lemma 2.5. If $H \subseteq G$ then $\kappa(H) \leq \kappa(G)$.

This is not true for the operation of contracting an edge. For instance, $\kappa\left(K_{2}\right)=1<\kappa\left(C_{3}\right)=3 / 2$, while $\kappa\left(C_{4}\right)<\kappa\left(C_{3}\right)=3 / 2$ (cf. Theorem 3.3). So $\kappa(G)$ and $\kappa(G / e)$ are not comparable in general.

Given two graphs $G_{1}=\left(V_{1}, E_{1}\right)$ and $G_{2}=$ $\left(V_{2}, E_{2}\right)$ for which $V_{1} \cap V_{2}$ is a clique in both $G_{1}$ and $G_{2}$, the graph $G=\left(V_{1} \cup V_{2}, E_{1} \cup E_{2}\right)$ is called their clique sum, or their clique $k$-sum when $\left|V_{1} \cap V_{2}\right|=k$.

Lemma 2.6. Assume $G$ is the clique $k$-sum of $G_{1}$ and $G_{2}, k \leq 3$. Then, $\kappa(G)=\max \left(\kappa\left(G_{1}\right), \kappa\left(G_{2}\right)\right)$.

Proof. Let $\lambda:=\max \left(\kappa\left(G_{1}\right), \kappa\left(G_{2}\right)\right)$ and $n=|V|$. The inequality $\kappa(G) \geq \lambda$ follows from Lemma 2.5.

For the other direction, let $x \in \mathcal{E}(G)$ and $X \in \mathcal{E}_{n}$ such that $x=\pi_{E}(X)$; we have to show that $x \in$ $\lambda \cdot \operatorname{CUT}(G)$. Let $X_{i}$ denote the principal submatrix of $X$ indexed by $V_{i}$, for $i=1,2$. As $\mathcal{E}\left(G_{i}\right) \subseteq \kappa\left(G_{i}\right)$. $\operatorname{CUT}\left(G_{i}\right) \subseteq \lambda \cdot \operatorname{CUT}\left(G_{i}\right)$, we deduce that $\pi_{E_{i}}\left(X_{i}\right) \in$ $\lambda \cdot \operatorname{CUT}\left(G_{i}\right)$. Since the linear inequality description of $\operatorname{CUT}(G)$ is obtained by juxtaposing the linear inequality descriptions of $\operatorname{CUT}\left(G_{1}\right)$ and $\operatorname{CUT}\left(G_{2}\right)$ and identifying the variables corresponding to edges contained in $V_{1} \cap V_{2}$ [4], the claim follows.

\section{Computing the Grothendieck constant for some graph classes}

We start this section by introducing the main objects and some fundamental results associated with them, that form the basic ingredients of our approach.

A graph $H$ is called a minor of a graph $G$, denoted by $H \preccurlyeq G$, if $H$ can be obtained from $G$, through a series of edge deletions and edge contractions.
Given a graph $G=([n], E)$, consider the metric polytope $\operatorname{MET}(G) \subseteq \mathbb{R}^{E}$ defined by the inequalities $-1 \leq x_{e} \leq 1$ for $e \in E$, and

$$
x(C \backslash F)-x(F) \leq|C|-2,
$$

for every circuit $C$ in $G$ and $F \subseteq C$ with $|F|$ odd [9]. Additionally, define $\operatorname{MET}^{01}(\bar{G}):=f(\operatorname{MET}(G))$, where $f(x)=e-2 x$ for $x \in \mathbb{R}^{E}$. The cut and metric polytopes are related as follows.

Theorem 3.1. [5] For any graph $G, \operatorname{CUT}(G) \subseteq$ $\operatorname{MET}(G)$, with equality if and only if $K_{5} \npreceq G$.

Any matrix $X \in \mathcal{E}_{n}$ has its diagonal entries all equal to 1 . Hence all its entries lie in $[-1,1]$ and can thus be parametrized as $x_{i j}=\cos \left(\pi y_{i j}\right)$ where $y_{i j} \in[0,1]$. Let $\cos \left(\pi \operatorname{MET}^{01}(G)\right)=$

$$
\left\{\left(\cos \left(\pi y_{e}\right)\right)_{e \in E} \mid y \in \operatorname{MET}^{01}(G)\right\} .
$$

Theorem 3.2. [14] $\mathcal{E}(G) \subseteq \cos \left(\pi \mathrm{MET}^{01}(G)\right)$, with equality if and only if $K_{4} \npreceq G$.

Thus, equality holds when $G=C_{n}$. Moreover,

Lemma 3.1. [6] For $p$ even, we have $c e^{T} \in \mathcal{E}\left(C_{p}\right)$ for all $c \in[-1,1]$. For $p$ odd, we have $c e^{T} \in \mathcal{E}\left(C_{p}\right)$ if and only if $-\cos \frac{\pi}{p} \leq c \leq 1$.

\subsection{The case of circuits}

Using the parametrizations of $\operatorname{MET}\left(C_{n}\right)$ and $\mathcal{E}\left(C_{n}\right)$ given by Theorems 3.1 and 3.2 respectively, we are able to compute $\kappa\left(C_{n}\right)$. Specifically,

Theorem 3.3. The Grothendieck constant of a circuit $C_{n}$ of length $n \geq 3$ is equal to

$$
\kappa\left(C_{n}\right)=\frac{n}{n-2} \cos \left(\frac{\pi}{n}\right) .
$$

Proof. By Lemma 2.2 it suffices to compute $\kappa\left(C_{n}, w\right)$ for facet defining inequalities of $\operatorname{CUT}\left(C_{n}\right)$. By Theorem 3.1, they correspond to the circuit inequalities and, since they are all switching equivalent, it suffices to consider one of them; for instance, we can choose $w^{T} x=-x(E)$ for odd $n$, and $w^{T} x=x_{e}-x(E \backslash\{e\})$ for even $n$. In both cases, we find that $\operatorname{ip}\left(C_{n}, w\right)=n-2$. Thus it now suffices to show that $\operatorname{sdp}\left(C_{n}, w\right)=n \cos (\pi / n)$ as this will give the desired value for $\kappa\left(C_{n}, w\right)$.

For $n$ odd, it is known that $\operatorname{sdp}_{\mathrm{GW}}\left(C_{n}, e\right)=$ $\frac{n}{4}\left(2+2 \cos \frac{\pi}{n}\right) \quad$ (see [19]), which implies that $\operatorname{sdp}\left(C_{n},-e\right)=2 \operatorname{sdp}_{\mathrm{GW}}\left(C_{n}, e\right)-n=n \cos (\pi / n)$. 
This can also be easily verified using the parametrization of $\mathcal{E}\left(C_{n}\right)$ from Theorem 3.2 .

One can also compute $\operatorname{sdp}\left(C_{n}, w\right)$ for $n$ even and $w=(-1,1, \ldots, 1)$ using Theorem 3.2 . it turns out that this has also been computed in 22] in the context of quantum information theory.

\subsection{The case of $K_{5}$-minor free graphs}

Since $K_{5}$-minor free graphs are 4-colorable [21], we deduce from (4) that their Grothendieck constant $\kappa(G)$ is bounded. Here we give a closed-form formula for $\kappa(G)$ in terms of the girth of $G$.

Theorem 3.4. If $G$ is a graph with no $K_{5}$ minor (and $G$ is not a forest), then

$$
\kappa(G)=\frac{g}{g-2} \cos \left(\frac{\pi}{g}\right),
$$

where $g$ is the minimum length of a circuit in $G$.

Proof. Directly from Theorem 3.3 using the facts that all facets of $G$ are supported by circuits (Theorem 3.1) and that the function $\frac{n}{n-2} \cos \left(\frac{\pi}{n}\right)$ is monotone nonincreasing in $n$.

As a direct application we recover the values $\kappa\left(K_{2, n}\right)=\kappa\left(K_{3, n}\right)=\sqrt{2}$, for $n \geq 3[10]$.

\subsection{Graphs whose cut polytope is defined by in- equalities supported by at most $k$ points}

We show here that the Grothendieck constant can be bounded in terms of the size of the supports of the inequalities defining facets of the cut polytope. The support graph of an inequality $w^{T} x \leq 1$ is the graph $H=(W, F)$, where $F=\left\{i j \in E \mid w_{i j} \neq\right.$ $0\}$ and $W$ is the set of nodes covered by $F$. We say that $w^{T} x \leq 1$ is supported by at most $k$ points when $|W| \leq k$. For instance, a triangle inequality is supported by three points.

Fix an integer $k \geq 2$. Let $\mathcal{R}_{k}\left(K_{n}\right) \subseteq \mathbb{R}^{E_{n}}$ be the polyhedron defined by all valid inequalities for $\mathrm{CUT}_{n}$ supported by at most $k$ points. For $G=$ $([n], E)$, let $\mathcal{R}_{k}(G):=\pi_{E}\left(\mathcal{R}_{k}\left(K_{n}\right)\right)$. For instance, $\mathcal{R}_{3}\left(K_{n}\right)=\operatorname{MET}\left(K_{n}\right)$, and thus $\mathcal{R}_{3}(G)=\operatorname{MET}(G)$.

Clearly, $\operatorname{CUT}(G) \subseteq \mathcal{R}_{k}(G)$. Define the class $\mathcal{G}_{k}$ of all graphs $G$ for which $\operatorname{CUT}(G)=\mathcal{R}_{k}(G)$. For instance, $\mathcal{G}_{2}$ consists of all forests (thus the $K_{3}$ minor free graphs) and $\mathcal{G}_{3}$ of the $K_{5}$-minor free graphs. Thus both are closed under taking minors; this holds for any $\mathcal{G}_{k}$.

Theorem 3.5. The class $\mathcal{G}_{k}$ is closed under taking minors.
ProOF. It follows directly from the definition that $\mathcal{G}_{k}$ is closed under edge deletion. It remains to verify that it is closed under edge contraction. Let $G=(V, E)$ and $G^{\prime}:=G / e=\left(V^{\prime}, E^{\prime}\right)$, where $e=(1,2)$ and $V^{\prime}=\{2, \ldots, n\}$.

Given $y \in \mathbb{R}^{E^{\prime}}$, define its extension $\tilde{y} \in \mathbb{R}^{E}$ by $\tilde{y}_{12}=1, \tilde{y}_{1 i}=y_{2 i}$ if $1 i \in E$ with $i \geq 3, \tilde{y}_{2 i}=y_{2 i}$ if $2 i \in E$ with $i \geq 3$, and $\tilde{y}_{i j}=y_{i j}$ if $i j \in E$ with $i, j \geq 3$. One can easily verify that $\tilde{y} \in \operatorname{CUT}(G)$ iff $y \in \mathrm{CUT}(G / e)$.

We now verify that if $y \in \mathcal{R}_{k}\left(K_{n-1}\right)$, then $\tilde{y} \in$ $\mathcal{R}_{k}\left(K_{n}\right)$. Indeed, say $w^{T} x \leq 1$ is a valid inequality for $\mathrm{CUT}_{n}$ supported by at most $k$ points. Define the inequality on $x=\left(x_{i j}\right)_{2 \leq i<j \leq n}$ :

$b^{T} x:=\sum_{i=3}^{n}\left(w_{1 i}+w_{2 i}\right) x_{2 i}+\sum_{3 \leq i<j \leq n} w_{i j} x_{i j} \leq 1-w_{12}$.

Obviously it is supported by at most $k$ points and it is valid for $\mathrm{CUT}_{n-1}$. Hence $b^{T} y \leq 1-w_{12}$, which implies $w^{T} \tilde{y} \leq 1$.

Assume $G \in \mathcal{G}_{k}$, i.e., $\operatorname{CUT}(G)=\pi_{E}\left(\mathcal{R}_{k}\left(K_{n}\right)\right)$ and let $z \in \pi_{E^{\prime}}\left(\mathcal{R}_{k}\left(K_{n-1}\right)\right)$; we show that $z \in$ $\operatorname{CUT}(G / e)$. Say $z=\pi_{E^{\prime}}(y)$ where $y \in \mathcal{R}_{k}\left(K_{n-1}\right)$. By the discussion above, the extension $\tilde{y}$ of $y$ belongs to $\mathcal{R}_{k}\left(K_{n}\right)$ and thus $\pi_{E}(y) \in \pi_{E}\left(\mathcal{R}_{k}\left(K_{n}\right)\right)=$ $\operatorname{CUT}(G)$. This in turn implies that $z \in \operatorname{CUT}(G / e)$ since $\pi_{E}(y)$ is the extension of $z$.

Clearly, for $G \in \mathcal{G}_{2}, \kappa(G)=\kappa\left(K_{2}\right)=1$. Moreover, Theorem 3.4 implies that $\kappa(G) \leq \kappa\left(K_{3}\right)=$ $3 / 2$ for $G \in \mathcal{G}_{3}$. This pattern extends to any $k$.

Theorem 3.6. If $G \in \mathcal{G}_{k}$ then $\kappa(G) \leq \kappa\left(K_{k}\right)$. Moreover, this bound is tight since $K_{k} \in \mathcal{G}_{k}$.

ProOF. It is enough to show that for any graph $G, \mathcal{E}(G) \subseteq \kappa\left(K_{k}\right) \cdot \mathcal{R}_{k}(G)$. Moreover, it suffices to consider only $G=K_{n}$, as the general result follows by taking projections.

Let $y \in \mathcal{E}\left(K_{n}\right)$ and let $w^{T} x \leq 1$ be a valid inequality for $\mathrm{CUT}_{n}$ with support $H=(W, F)$ where $|W| \leq k$. Then, $w^{T} y=\pi_{F}(w)^{T} \pi_{F}(y) \leq$ $\operatorname{sdp}\left(H, \pi_{F}(w)\right) \leq \kappa(H) \cdot \operatorname{ip}\left(H, \pi_{F}(w)\right) \leq \kappa\left(K_{k}\right)$, where we use the facts that $\kappa(H) \leq \kappa\left(K_{k}\right)$ and $\operatorname{ip}\left(H, \pi_{F}(w)\right) \leq 1$ for the right most inequality.

One can verify that $\kappa\left(K_{7}\right)=3 / 2$ (see [15]). Hence, $\kappa(G) \leq 3 / 2$ for all $G \in \mathcal{G}_{7}$.

\section{Integrality gap of clique-web inequalities}

We have already seen that $\kappa\left(K_{n}\right)=\Theta(\log n)$ and it is an interesting question to identify explicit instances that achieve this integrality gap. This was 
posed as an open question in [1] and instances with large gap are given in [3]. In this section we show that the gap is bounded by 3 for clique-web inequalities, a wide class of valid inequalities for $\mathrm{CUT}_{n}$.

Given integers $p$ and $r$ with $p \geq 2 r+3$, the antiweb graph $\mathrm{AW}_{p}^{r}$ is the graph with vertex set $[p]$, and with edges $(i, i+1), \ldots,(i, i+r)$ for $i \in[p]$, where the indices are taken modulo $p$. The web graph $\mathrm{W}_{p}^{r}$ is defined as the complement of $\mathrm{AW}_{p}^{r}$ in $K_{p}$. Call the set of edges $(i, i+s)$ for $i \in[p]$ (indices taken modulo $p$ ) the $s$-th band, so that $\mathrm{AW}_{p}^{r}$ consists of the first $r$ bands and $\mathrm{W}_{p}^{r}$ consists of the last $\lceil q / 2\rceil$ bands in $K_{p}$.

Let $p, q, r, n$ be integers satisfying $p-q=2 r+1$, $q \geq 2, n=p+q$. The (pure) clique-web inequality with parameters $n, p, q, r$ is the inequality

$$
-x\left(K_{q}\right)-\sum_{\substack{1 \leq i \leq q \\ q+1 \leq j \leq n}} x_{i j}-x\left(\mathrm{~W}_{p}^{r}\right) \leq q(r+1) .
$$

The support graph of (5), denoted by $\mathrm{CW}_{p}^{r}$, consists of a clique on the first $q$ nodes, a web on the last $p$ ones, and a complete bipartite graph between them. It is known that clique-web inequalities define facets of $\mathrm{CUT}_{n}$. Note that hypermetric and bicycle odd wheel inequalities arise as special cases of (5), for $r=0$ and $r=\frac{n-5}{2}$, respectively (see [9]).

Since the left-hand side of (5) is equal to $-x\left(K_{p+q}\right)+x\left(\mathrm{AW}_{p}^{r}\right)$, one easily obtains that $\operatorname{sdp}\left(\mathrm{CW}_{p}^{r},-e\right) \leq(p+q) / 2+p r=q(r+1)+(2 r+$ $1)^{2} / 2$, which implies that $\kappa\left(\mathrm{CW}_{p}^{r},-e\right) \leq 1+\frac{(2 r+1)^{2}}{q(2 r+2)}$. This directly implies the following:

Lemma 4.1. The integrality gap of a clique-web inequality with $q \geq 2 r+1$ is upper bounded by 2 .

We now consider the case when $q \leq 2 r$.

Theorem 4.1. The integrality gap of a clique-web inequality with $q \leq 2 r$ is upper bounded by 3 .

PRoOF. We can rewrite $\operatorname{sdp}\left(\mathrm{CW}_{p}^{r},-e\right)$ as

$$
\max _{X \in \mathcal{E}_{n}}-\sum_{i j \in K_{q}} X_{i j}-\sum_{\substack{1 \leq i \leq q \\ q+1 \leq j \leq n}} X_{i j}-\sum_{i j \in \mathrm{W}_{p}^{r}} X_{i j} .
$$

Notice that the program (6) is invariant under the action of the full symmetric group $S_{q}$ acting on the row/column indices in $[q]$. Moreover, (6) is invariant under the action of the group of cyclic permutations in $S_{p}$ acting on the row/column indices in $\{q+1, . ., n\}$. Thus, we can restrict without loss of generality the matrix $X$ in (6) to satisfy the following invariance conditions:

$$
\begin{array}{ll}
X_{i j}=a & \text { for } 1 \leq i \neq j \leq q, \\
X_{i j}=b & \text { for } 1 \leq i \leq q<j \leq n, \\
X_{i j}=c_{|j-i \bmod p|} & \text { for } q+1 \leq i \neq j \leq n
\end{array}
$$

for some scalars $a, b, c_{1}, \ldots, c_{\lceil q / 2\rceil}$. Hence $X$ has the form $X:=\left(\begin{array}{cc}a J_{q, q}+(1-a) I_{q} & b J_{q, p} \\ b J_{p, q} & X_{p}\end{array}\right)$, where $X_{p}$ denotes the principal submatrix of $X$ indexed by $\{q+1, \ldots, n\}$. One can easily verify that $X \succeq 0$ if and only if $Y:=\left(\begin{array}{cc}\beta & b e^{T} \\ b e & X_{p}\end{array}\right) \succeq 0$, after setting $\beta:=\frac{(q-1) a+1}{q}$.

Consider first the case when $q$ is even; so $p$ is odd, all bands in $\mathrm{W}_{p}^{r}$ have size $p$, and the objective function in (6) reads

$$
\frac{q}{2}(1-q \beta)-p q b-p\left(c_{1}+\ldots+c_{q / 2}\right) .
$$

If $\beta=0$, then $b=0$ and Lemma 3.1 implies that $c_{s} \geq-\cos (\pi / p)$ for all $s$. Indeed each band of $\mathrm{W}_{p}^{r}$ is a circuit or a disjoint union of circuits (e.g. the first band of $\mathrm{W}_{9}^{2}$ is a union of three triangles). As $p$ is odd, at least one of these circuits is an odd circuit of size $p^{\prime} \leq p$, so that Lemma 3.1 implies that the entries on the band are at least $-\cos \left(\pi / p^{\prime}\right) \geq-\cos (\pi / p)$. Now the objective value is equal to $\frac{q}{2}-p\left(c_{1}+\ldots+c_{q / 2}\right) \leq \frac{q}{2}(p \gamma+1) \leq$ $\frac{q}{2}(p+1)=\frac{q}{2}(q+2 r+2) \leq 2 q(r+1)($ as $q \leq 2 r)$, setting $\gamma:=\cos (\pi / p)$.

Assume now $\beta>0$. Taking the Schur complement in the above matrix $Y$ with respect to the entry $\beta$, we can rewrite the condition $Y \succeq 0$ as $X_{p}-\frac{b^{2}}{\beta} J \succeq 0$. If $\beta=b^{2}$, then $c_{s}=1$ for all $s$ and the maximum of $\frac{q}{2}\left(1-q b^{2}\right)-p q b-p q / 2$ for $b \in[-1,1]$ is easily verified to be equal to $q(r+1)$, attained at $b=-1$. Now let $\beta>b^{2}$ and $X \succeq 0$ is equivalent to $Z:=\frac{\beta}{\beta-b^{2}} X_{p}-\frac{b^{2}}{\beta-b^{2}} J \in \mathcal{E}_{p}$. As above, Lemma 3.1 permits to bound the entries of $Z$ as follows: $\frac{\beta}{\beta-b^{2}} c_{s}-\frac{b^{2}}{\beta-b^{2}} \geq-\gamma$ for $1 \leq s \leq q / 2$. Therefore, the program (6) is upper bounded by

$$
\begin{array}{ll}
\max _{b, c, \beta} & \frac{q}{2}(1-q \beta)-p q b-c p q / 2 \\
\text { s.t. } & \beta(c+\gamma) \geq b^{2}(\gamma+1) \\
& b^{2}<\beta \leq 1,-1 \leq b, c \leq 1
\end{array}
$$

At optimality, equality $\beta(c+\gamma)=b^{2}(\gamma+1)$ holds. This permits to express $c$ in terms of $b, \beta$ and to rewrite the objective function of (8) as $\frac{q}{2}(1-q \beta)-$ 
$\frac{p q}{2}\left(b^{2} \frac{\gamma+1}{\beta}+2 b-\gamma\right)$. For fixed $\beta$, the maximum of this quadratic function in $b$ is attained at $b=$ $-\frac{\beta}{\gamma+1} \in[-1,1]$ and is equal to $\frac{q}{2}(1-q \beta)+\frac{p q}{2}\left(\frac{\beta}{\gamma+1}+\right.$ $\gamma)=\frac{q}{2}\left(\beta\left(\frac{p}{\gamma+1}-q\right)+p \gamma+1\right)$. As $q \leq 2 r$, we have $\frac{p}{\gamma+1} \leq q$ and thus the latter quantity is maximized when $\beta=1$, so that the maximum of (8) is equal to $\frac{p q}{2}\left(\gamma+\frac{1}{\gamma+1}\right)-\frac{q(q-1)}{2}$. Hence, using $q \leq 2 r$ and $\gamma+\frac{1}{\gamma+1} \leq \frac{3}{2}$, we deduce that this maximum is upper bounded by $3 q(r+1)$. This concludes the proof that the integrality gap of the clique-web inequality is at most 3 when $q$ is even.

Consider now the case when $\mathrm{q}$ is odd. Then $p$ is even and $\mathrm{W}_{p}^{r}$ consists of $(q-1) / 2$ bands of size $p$ and one band of size $p / 2$. The treatment is analogous to the case $q$ even, except we must replace the objective function in (7) by

$$
\frac{q}{2}(1-q \beta)-p q b-p\left(c_{1}+\ldots+c_{\frac{q-1}{2}}\right)-\frac{p}{2} c_{\frac{q+1}{2}}
$$

and, as $p$ is even, the values on the bands can only be claimed to lie in $[-1,1]$ by Lemma 3.1 (which amounts to setting $\gamma=1$ in the above argument). Specifically, if $\beta=0$ we can upper bound the objective function (9) by $2 q(r+1)$ and, if $\beta=b^{2}$, we can upper bound (9) by $q(r+1)$. Finally, if $\beta>b^{2}$, as above we do a Schur complement and obtain $\frac{\beta}{\beta-b^{2}} c_{s}-\frac{b^{2}}{\beta-b^{2}} \geq-1$, so that (9) is upper bounded by the program (8) setting there $\gamma=1$. Hence the integrality gap of the clique-web inequality is also bounded by 3 for $q$ odd.

We conclude with several remarks.

- We just showed: (i) $\kappa\left(\mathrm{CW}_{r}^{p},-e\right) \leq 1+\frac{(2 r+1)^{2}}{q(2 r+2)}$, and (ii) $\kappa\left(\mathrm{CW}_{r}^{p},-e\right) \leq \frac{q+6 r+5}{4(r+1)}$ when $q \leq 2 r$. Therefore, asymptotically, the integrality gap tends to 1 as $q \rightarrow \infty$ and $r$ is fixed (by (i)), and it tends to $3 / 2$ as $r \rightarrow \infty$ and $q$ is fixed (by (ii)).

- Our analysis is tight for $q=2$, the case of bicycle odd wheel inequalities (since then the program (8) is equivalent to (6) $)$; that is, $\operatorname{sdp}\left(\mathrm{CW}_{2 r+3}^{r},-e\right)=$ $-1+p\left(\cos (\pi / p)+\frac{1}{\cos (\pi / p)+1}\right)$ (as mentioned in [19]). This explains why in the proof of Theorem 4.1 we use the precise estimate $-\cos \frac{\pi}{p} \leq c$, as opposed to the trivial bound $-1 \leq c$, which was equally good for the our asymptotic analysis.

- Pitowsky [17] shows the asymptotic lower bound $4 / \pi \sim 1.27$ for the integrality gap of the clique-web inequality with $q=2 r$ and $r \rightarrow \infty$.

- Given $b \in \mathbb{Z}^{n}$ with $\sum_{i} b_{i}=1$, the hypermetric inequality $w^{T} x:=-\sum_{1 \leq i<j \leq n} b_{i} b_{j} x_{i j} \leq\left(\sum_{i} b_{i}^{2}-1\right) / 2$ satisfies $\operatorname{sdp}\left(K_{n}, w\right) \leq \sum_{i} b_{i}^{2} / 2$; thus its integrality gap is at most $3 / 2$, with equality if and only if $b=(1,1,-1,0, \ldots, 0)$ (the case of triangle inequalities).

\section{References}

[1] N. Alon, K. Makarychev, Y. Makarychev, and A. Naor. Quadratic forms on graphs. Inventiones Mathimaticae, 163(3):499-522, 2006

[2] N. Alon and A. Naor. Approximating the cut-norm via Grothendieck's inequality. In STOC, pages 72-80, 2004.

[3] S. Arora, E. Berger, G. Kindler, M. Safra, and E. Hazan. On non-approximability for quadratic programs. In FOCS, pages 206-215, 2005.

[4] F. Barahona. The max-cut problem on graphs not contractible to $K_{5}$. Oper. Res. Lett., 2(3):107-111, 1983.

[5] F. Barahona and A. Mahjoub. On the cut polytope. Math. Program., 36:157-173, 1986.

[6] W.W. Barrett, C.R. Johnson, and P. Tarazaga. The real positive definite completion problem for a simple cycle. Linear Algebra Appl., 192:3-31, 1993.

[7] M. Braverman, K. Makarychev, Y. Makarychev, and A. Naor. The Grothendieck constant is strictly smaller than Krivine's bound. Preprint, arXiv:1103.6161 2 .

[8] J. Briët, F. de Oliveira Filho, and F. Vallentin. Grothendieck inequalities for semidefinite programs with rank constraints. Preprint, arXiv:1011.1754 1.

[9] M.M. Deza and M. Laurent. Geometry of Cuts and Metrics. Springer, 1997.

[10] P.C. Fishburn and J.A. Reeds. Bell inequalities, Grothendieck's constant, and root two. SIAM J. Disc. Math.,7(1):48-56,1994.

[11] M.X. Goemans and D.P. Williamson. Improved approximation algorithms for maximum cut and satisfiability problems using semidefinite programming. J. ACM, 42:1115-1145, 1995.

[12] A. Grothendieck. Résumé de la théorie métrique des produits tensoriels topologiques. Bol. Soc. Mat. Sao Paolo., 8:1-79, 1953.

[13] J. Krivine. Sur la constante de Grothendieck. C. R. Acad. Sci. Paris Sér. A-B, 284(8):A445A446, 1977.

[14] M. Laurent. The real positive semidefinite completion problem for series-parallel graphs. Linear Algebra Appl., 252:347-366, 1997.

[15] M. Laurent. Semidefinite relaxations for Max-Cut. In The Sharpest Cut: The Impact of Manfred Padberg and His Work. M. Grötschel, ed., pages 257-290, MPS-SIAM Series in Optimization 4, 2004.

[16] L. Lovász. On the Shannon capacity of a graph. IEEE Trans. Inform. Th., IT-25:1-7, 1979.

[17] I. Pitowsky. New Bell inequalities for the singlet state: Going beyond the Grothendieck bound. Journal of Mathematical Physics, 49:012101, 2008.

[18] S. Poljak. C. Delorme. Combinatorial properties and the complexity of a max-cut approximation. European J. Combin., 14:313-333, 1993.

[19] S. Poljak. C. Delorme. The performance of an eigenvalue bound on the max-cut problem in some classes of graphs. Discrete Math., 111:145-156, 1993.

[20] J.A. Reeds. A new lower bound on the real Grothendieck constant. Preprint, 1991. Available at http://www.dtc.umn.edu/ reedsj/bound2.dvi 
[21] K. Wagner. Über eine Eigenschaft der ebenen Komplexe. Math. Ann., 114(1):570-590, 1937.

[22] S. Wehner. Tsirelson bounds for generalized ClauserHorne-Shimony-Holt inequalities. Phys. Rev. A, 73:022110, 2006. 